\title{
Dynamic Role of the Correlation Effect Revealed in the Exceptionally Slow Autodetachment Rates of the Vibrational Feshbach Resonances in the Dipole-Bound State
}

\author{
Do Hyung Kang, Jinwoo Kim and Sang Kyu Kim* \\ Department of Chemistry, KAIST, Daejeon 34141, Republic of Korea \\ *Corresponding Author: sangkyukim@kaist.ac.kr
}

\begin{abstract}
Real-time autodetachment dynamics of the loosely-bound excess electron from the vibrational Feshbach resonances of the dipole-bound states (DBS) of 4-bromophonoxide (4-BrPhO-) and 4-chlorophenoxide (4$\mathrm{ClPhO}^{-}$) anions have been thoroughly investigated. The state-specific autodetachment rate measurements obtained by the picosecond time-resolved pump-probe method on the cryogenically cooled anions, exhibit the exceptionally long lifetime $(\tau)$ of $\sim 2.5 \pm 0.6 \mathrm{~ns}$ (as the upper bound) for the $11^{\prime 1}$ vibrational mode of the 4-BrPhO- DBS. Strong mode-dependency in the wide dynamic range has also been found, giving $\tau \sim 5.3 \mathrm{ps}$ for the $10^{\prime 1}$ mode, for instance. Though it is nontrivial to get the state-specific rates for the 4-ClPhO- DBS, the average autodetachment lifetime of the $19^{\prime 1} 20^{\prime 1} / 11^{\prime 1}$ mode has been estimated to be $\sim 548 \pm 108$ ps. Observation of these exceptionally slow autodetachment rates of vibrational Feshbach resonances strongly indicates that the 'correlation effect' may play a significant role in the DBS photodetachment dynamics. The Fermi's golden rule has been invoked so that the correlation effect is taken into account in the form of the interaction between the charge and the induced dipole where the latter is given by the polarizable counterparts of the electron-rich halogenated compound and the diffuse non-valence electron. This report suggests that one may measure, from the real-time autodetachment dynamics, the extent of the correlation effect contribution to the stabilization and/or dynamics of the excess non-valence electron among many different types of the long-range interactions of the DBS.
\end{abstract}


Since firstly conceived by Fermi and Teller, ${ }^{1}$ the dipole-bound state (DBS) of the anion, where the excess electron is loosely bound to the neutral core by the long-range monopole-dipole interaction, has been intensively investigated both experimentally and theoretically for many recent decades. $^{2-8}$ The DBS is known to play an important role as the doorway state to the stable valence anion formation. ${ }^{9-13}$ Namely, as the slow electron approaches the neutral molecule or radical, the incoming electron is captured in the form of the Feshbach resonances by the long-range attractive interaction potential, and it is followed by the subsequent coupling and/or relaxation into the more stable anion species. ${ }^{14,15}$ Detailed pictures of the whole processes of the electron-capturing, coupling, and relaxation are thus quite essential for the thorough understanding of the anion chemistry as well as the entry/exit dynamics of the redox reactions. The DBS has been found to be ubiquitous and identified in a number of chemical and biological systems to date. Notably, thanks to the combined techniques of the laser and cryogenically cooled ion-trap, ${ }^{16}$ the understanding of the DBS has recently been enormously advanced in terms of the precise information of energetics, vibrational structures, and state-specific autodetachment (or relaxation) dynamics.5, 17 Regarding dynamics, however, the state-specific autodetachment rate of the DBS was directly measured only recently for the phenoxide (PhO-) anion, ${ }^{18}$ allowing for the stringent comparison of the experiment with the theoretical prediction. The Fermi's golden rule has been found to be extremely useful to explain the strongly mode-dependent autodetachment rates although the prediction of the absolute values seems to be still quite challenging. ${ }^{18}$

The lower threshold of the dipole moment for holding the excess electron was firstly proposed to be $1.625 \mathrm{D},{ }^{19}$ although it has been refined repeatedly after the correction of the BornOppenheimer approximation, ${ }^{20,21}$ for instance. More practically, however, it seems to be widely accepted now, as a rule of thumb, that the DBS may exist when the dipole moment of the neutral core exceeds 2.5 D. ${ }^{22,} 23$ Although the long-range interaction of the dipole moment of the neutral core with the excess non-valence electron has been considered to be the most critical factor in the electron binding/unbinding dynamics, many theoretical works have suggested that the (especially dispersive) electron correlation effect should be largely responsible for the excess 
electron binding to the neutral core in the DBS., 24-26 The significant contribution of the electron correlation effect to the binding energy of DBS has been theoretically demonstrated from the quantum-mechanical calculations using the $2^{\text {nd-order }}$ Møller-Plesset perturbation (MP2), the coupled cluster singles and doubles (CCSD) theory, or the quantum Monte Carlo method. ${ }^{27-30}$ In the same context, it is notable that the critical value of the dipole-moment for the existence of the $\pi$-type DBS is still in dispute. ${ }^{31-33}$ Although the extent of the correlation effect is highly anticipated to be strongly dependent on individual chemical systems, the importance of the correlation effect in the DBS seems to be well received in the scientific community. Apparently, however, it is nontrivial to experimentally identify the correlation effect in terms of the static and/or dynamic role in the DBS. Even though the electron binding energy of the DBS is often expected to be proportional to the dipole-moment magnitude of the neutral core, it does not necessarily mean that the electron binding of the DBS is governed by the dipole moment only, as many different factors related to the long-range interaction potential could be cancelled out or added up depending on the chemical details. ${ }^{34}$ For example, the smaller (or lager) binding energy does not necessarily mean the smaller (or larger) contribution of the correlation effect, and vice versa.

In this aspect, we have here found that the correlation effect may be reflected in the dynamic property of the DBS rather than in the static binding energy. For example, two different DBS chemical systems of the similar binding energies could be quite different in terms of the extent of the correlation-effect. Herein, we argue that the autodetachment dynamics could reveal the nature of the electron binding in terms of the dynamic role of the correlation effect in the electron binding/unbinding dynamics of the DBS. We have investigated the picosecond (ps) timeresolved autodetachment dynamics of the DBS vibrational Feshbach resonances prepared by the one-photon photoexcitation of the cryogenically-cooled 4-bromophenoxide (4-BrPhO-) and 4chlorophenoxide (4-ClPhO-) anions. Exceptionally slow autodetachment dynamics observed in some vibrational Feshbach resonances of these anions have been analyzed from the new perspective that the correlation effect may play a significant role in the autodetachment dynamics. 

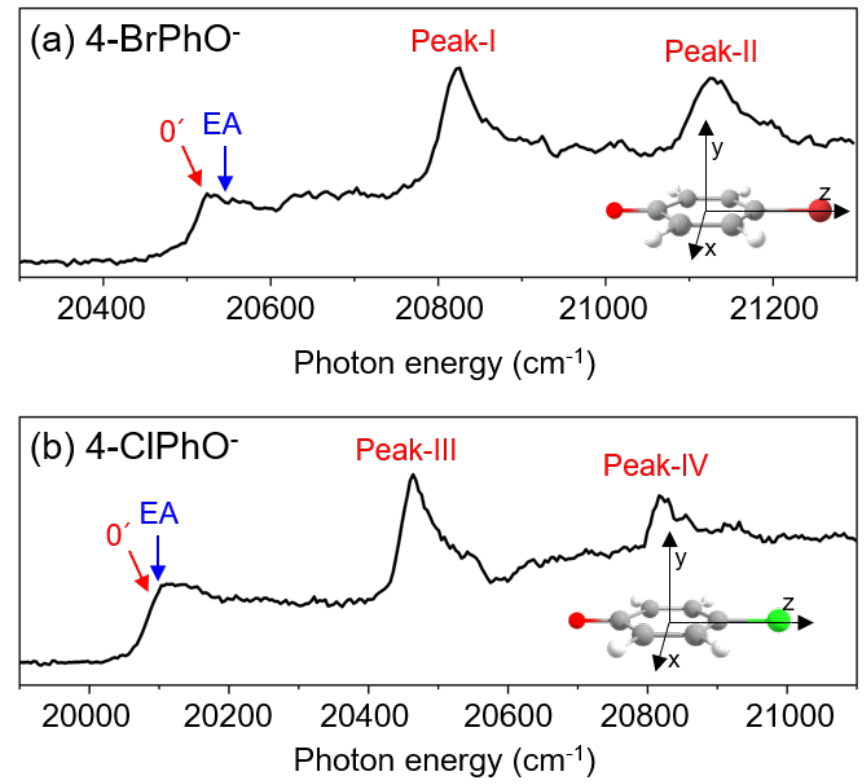

Figure 1. Picosecond photodetachment spectra of the (a) 4-BrPhO- and (b) 4-ClPhO- Vibrational states of the DBS are labeled in red while the electron affinity (EA) of each anion is labeled by the blue arrow. Cartesian coordinates of each anion are depicted in the insets.

Photodetachment spectra of the cryogenically-cooled 4-BrPhO- and 4-ClPhO- anions taken by monitoring the total photoelectron signal as a function of the pump laser wavelength are shown in Figure 1. In both spectra, the stepwise increases of the photoelectron signal represent the electron-affinity (EA) thresholds. The rather sharp peaks are attributed to the vibrational Feshbach resonances of the DBS whereas the diffusive structureless background signal originates from the direct photodetachment of the anion. The overall structures of the photodetachment spectra are more or less identical to those obtained by the nanosecond (ns) laser pulse reported by the Wang group ${ }^{22}$ except the broad bandwidths of the vibrational bands due to the intrinsic property of the picosecond (ps) laser pulse $\left(\Delta E \sim 20 \mathrm{~cm}^{-1}, \Delta t \sim 1.7 \mathrm{ps}\right)$. The binding energy of the DBS has been precisely estimated to be 24 or $11 \mathrm{~cm}^{-1}$ for $4-\mathrm{BrPhO}^{-}$or $4-\mathrm{ClPhO}$, respectively. ${ }^{22}$ The zero-point energy (ZPE) level of the DBS is hardly identified, as the ps laser bandwidth is

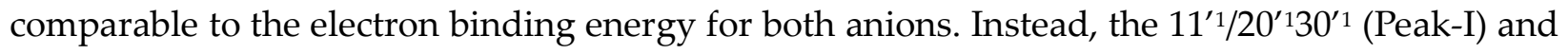
$11^{\prime 2} / 10^{\prime 1}$ (Peak-II) bands could be well identified for 4-BrPhO-, whereas the $19^{\prime 12} 20^{\prime 1} 11^{\prime 1}$ (Peak-III) 
and $11^{\prime 1} 19^{\prime} 120^{\prime 1} / 11^{\prime 2}$ (Peak-IV) bands stand out in the DBS of 4-ClPhO-, Figure 1. Compared to the previously reported highly-resolved spectra, the individual DBS vibrational modes could not be spectroscopically resolved in the broad bands in this work.

Now, by employing the ps pump-probe scheme, we could measure the state-specific autodetachment rates of vibrational Feshbach resonances for both 4-BrPhO- and 4-ClPhO-, Figure 2. The autodetachment rate has been determined by the transient taken by monitoring the low kinetic energy electron as a function of the delay-time between the pump and probe laser pulses. The pump laser wavelength is tuned at the particular DBS vibrational band while the spatially overlapped non-resonant probe laser pulse $(791 \mathrm{~nm})$ is given at different delay times. At the zero delay-time, the DBS is most efficiently depopulated to give a spike ${ }^{18}$ with the pump-probe crosscorrelation width of $\sim 2.88$ ps. With the increase of the pump-probe delay, the transient signal shows the apparent recovery (which is equivalent to the decay in the transients shown in Figure 2) due to the autodetachment process, giving the lifetime of the DBS Feshbach resonance from the exponential fit to the experiment.

The Peak-I transient of 4-BrPhO- shows the biexponential behavior with two distinct lifetimes, Figure 2. The faster decaying component gives the lifetime $(\tau)$ of $\sim 13.5 \pm 7.0$ ps whereas the lifetime of the slow-decaying component is found to be extremely long, giving $\tau \sim 2.54 \pm 0.60$ $\mathrm{ns}$ (as the upper bound) with the relative amplitude ratio of 0.43 to 0.57 , respectively. As the fundamental $11^{\prime 1}$ and $20^{\prime 1} 30^{\prime 1}$ combinational modes are expected to be co-excited within the ps pump laser spectral window, two distinct lifetimes are ascribed to the autodetachment of two different vibrational modes. For the appropriate matches between the individual vibrational modes and their associated lifetimes, the velocity-map electron image taken at the pump wavelength of the DBS resonance is compared with that taken at the adjacent non-resonant (allowing just the direct photodetachment) pump wavelength. The image of the former minus that of the latter then gives the nature of the DBS band as the propensity rule of $\Delta v=-1$ is rather strictly obeyed. Accordingly, in the photoelectron spectrum taken from the peak-I, the - $v_{11}$ peak 
is the consequence from the autodetachment of the $11^{\prime 1}$ mode whereas the $-v_{20}$ or $-v_{30}$ peak results from that of the $20^{\prime 1} 30^{\prime 1}$ combination band via the wobbling motion associated with the $v_{20}$ or $v_{30}$ mode, respectively. The relative contribution of the $11^{\prime 1}$ or $20^{\prime 130^{\prime 1}}$ band is then, from the comparison of integrated photoelectron peak areas, estimated to be 0.64 or 0.36 , respectively (Supporting Information). From the fact that the $11^{\prime 1}$ mode is more responsible for the peak-I, therefore, the exceptionally long lifetime of 2.54 ns should be due to the autodetachment from the $11^{\prime 1}$ mode whereas the faster decaying component $(\tau \sim 13.5 \mathrm{ps})$ is attributed to the autodetachment from the $20^{\prime 13} 0^{\prime 1}$ combinational mode. While the autodetachment lifetime of $13.5 \mathrm{ps}$ for the $20^{\prime} 130^{\prime 1}$ mode sounds reasonable in terms of the order of magnitudes, the lifetime of $2.54 \mathrm{~ns}$ for the $11^{\prime 1}$ mode seems to be extraordinarily long for the vibrational autodetachment process. It should be noted though that the estimated lifetime of 2.54 ns has the somewhat large uncertainty due to the narrow temporal window $(0-1.8 \mathrm{~ns})$ of the present experimental condition. Nonetheless, it is quite remarkable that the autodetachment rate of the $11^{\prime 1}$ mode of the $4-\mathrm{BrPhO}-\mathrm{DBS}$ is $\sim 75$ (or less) times slower compared to that of the $11^{\prime 1}$ mode of the $\mathrm{PhO}^{-}$of which the lifetime has been measured to be $~ 33.5 \mathrm{ps}^{18}$ Considering that the infrared intensity of the $v_{11}$ mode of the $4-\mathrm{BrPhO}$ is only two times weaker than the $v_{11}$ mode of the PhO (vide infra), the retardation of the DBS autodetachment of the former compared to the latter by nearly two orders of magnitudes is quite exceptional. The similar analysis for the $11^{\prime 2} / 10^{\prime 1}$ band (Peak-II) of 4-BrPhO- have been carried out (Supporting Information), giving $\tau \sim 82$ or 5.3 ps for the $11^{\prime 2}$ or $10^{\prime 1}$ mode, respectively. The autodetachment rate of the $11^{\prime 2}$ mode of $4-\mathrm{BrPhO}^{-}(\tau \sim 82 \mathrm{ps})$ is also quite slow compared to that of the $11^{\prime 2}$ mode of $\mathrm{PhO}^{-}(\tau \sim 12 \mathrm{ps}),{ }^{18}$ supporting the experimental finding of the extremely slow autodetachment rate for the $11^{\prime 1}$ mode of $4-\mathrm{BrPhO}^{-}$. 
(a) $4-\mathrm{BrPhO}^{-}$

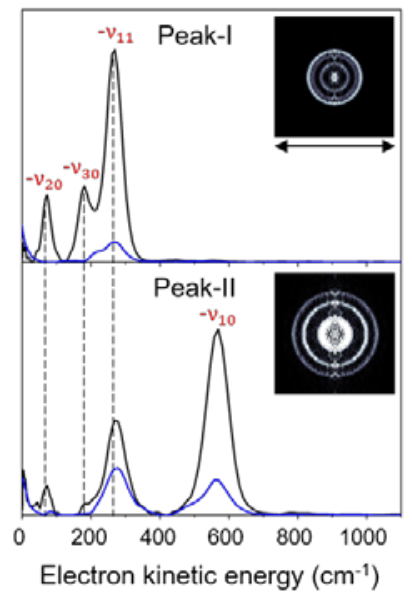

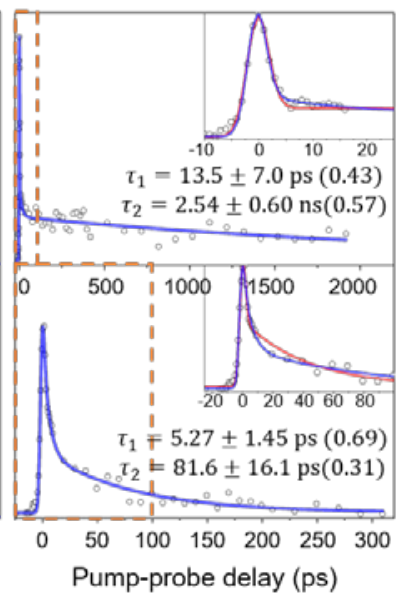

(b) $4-\mathrm{CIPhO}$

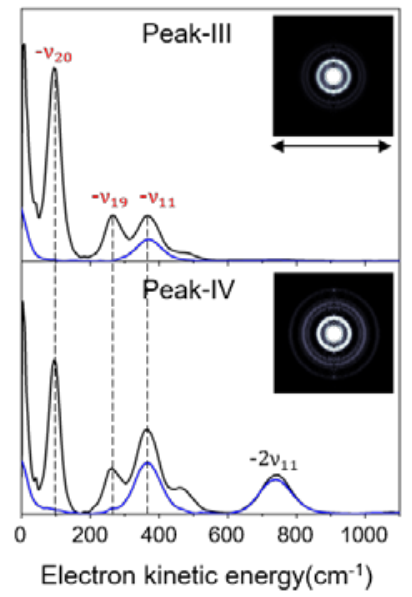

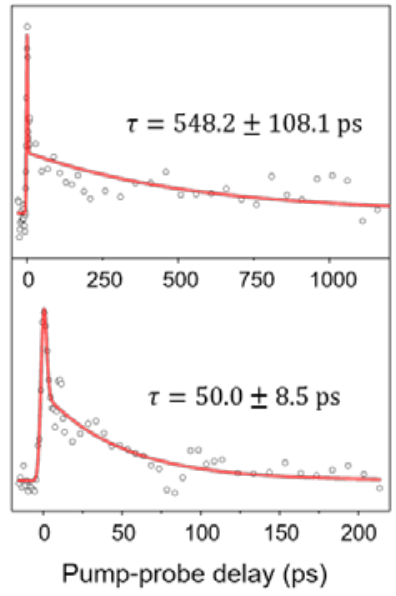

Figure 2. Photoelectron spectra and time-resolved photoelectron transients of the (a) 4-BrPhO- and (b) 4-ClPhO- DBS. (Left panels in (a) and (b)) Photoelectron spectra (black) taken at individual vibrational Feshbach resonances are compared with those taken at the adjacent non-resonant pump wavelengths (blue). Raw spectra of the latter are shifted by the amount of the photon energy difference for the purpose of the comparison. Largely-enhanced photoelectron peaks at the resonant excitations are appropriately labeled (red) by the vibrational modes responsible for the autodetachment. Re-constructed photoelectron images at the vibrational resonances are shown in the insets. The pump laser polarization axis is denoted as a black arrow. (Right panels in (a) and (b)) Picosecond time-resolved photoelectron transients taken at individual vibrational resonances are fitted by either the single (red) or bi-exponential (blue) decay function. Time constants are extracted from the most reliable fits. For 4-BrPhO, two distinct time constants and the relative amplitude ratios are obtained from the bi-exponential fits (see the text). Both single and bi-exponential fits are shown in the insets for 4-BrPhO.

In order to explain the experiment, we have invoked the Fermi's golden rule which has been widely used for the autodetachment rate..$^{35-38}$

$$
\begin{gathered}
k=\frac{2 \pi}{\hbar}\left|\left\langle\phi_{f}|W| \phi_{i}\right\rangle\right|^{2} \rho\left(K E_{e}\right) \\
\left\langle\phi_{f}|W| \phi_{i}\right\rangle=-\frac{\hbar^{2}}{2 m}\left\langle v_{f}\left|F(Q) \frac{\partial}{\partial Q}\right| v_{i}\right\rangle \\
\mathrm{F}(\mathrm{Q})=\frac{1}{\epsilon_{i f}}\left\langle e_{f}\left|\left(\frac{\partial U}{\partial Q}\right)\right| e_{i}\right\rangle
\end{gathered}
$$


Here, $\phi_{i}$ and $\phi_{f}$ are the initial and final total wavefunctions, respectively, whereas $v_{i}\left(e_{i}\right)$ or $v_{f}\left(e_{f}\right)$ is the initial or final vibrational (electronic) wavefunction, respectively. $\rho$ is the density of states which is the function of the electron kinetic energy $\left(K E_{e}\right) . \mathrm{U}$ is the charge-dipole interaction potential for the excess electron whereas $Q$ is the normal mode coordinate associated with the particular vibrational mode. When the electron binding potential is confined to the interaction between the charge and permanent-dipole moment $\left(\mu_{0}\right), F(Q)$ is proportional to the magnitude of the derivative of $\mu_{0}$ with respect to $Q, \frac{\partial \mu_{0}}{\partial Q}$. As the infrared (IR) intensity is proportional to $\left(\frac{\partial \mu_{0}}{\partial Q}\right)^{2}$, it is approximately regarded as the quantitative measure for the relative autodetachment rate of the corresponding vibrational mode. ${ }^{35}, 39$ Actually, the mode-dependent behavior of the autodetachment rate of the PhO- DBS could be quite successfully explained by the relative IR intensities of the individual vibrational modes as well as the Franck-Condon derivative factor for the overtone band. ${ }^{18}$ In this regard, the nearly two-orders of magnitudes increase of the lifetime of the $11^{\prime 1}$ mode of $4-\mathrm{BrPhO}^{-}$compared to that of the $11^{\prime 1} \mathrm{PhO}^{-}$mode cannot be explained by the simple application of the above conventional Fermi's golden rule, especially as the IR intensity of the former is only two times weaker than that of the latter (vide supra).

It should be emphasized that the autodetachment rate is little influenced by the amount of the electron-binding energy. Rather, the loosely-bound electron is shaken off by the dynamic change of the interaction potential induced by the vibrational wobbling motion. ${ }^{40}$ In this regard, one may invoke the aforementioned electron correlation effect into the autodetachment dynamics for the explanation of the large discrepancy of the experiment from the conventional Fermi's golden rule, especially as the electron-rich halogen atomic moiety is expected to be strongly correlated with the non-valence electron at the positive end of the dipole. Instead of the quantummechanical Hamiltonian, we have brought a simple physical model where the interaction potential in the Fermi's golden rule is modified to include the interaction between the charge and the (newly-added) induced dipole moment $\left(\vec{\mu}_{\text {ind }}\right)$. The effective dipole moment $\left(\vec{\mu}_{e f f}\right)$ is then the sum of the permanent and the induced dipole-moments. The induced dipole moment can be 
expressed by the relation of $\vec{\mu}_{\text {ind }}=\ddot{\alpha} \vec{E}$, where $\ddot{\alpha}$ is the polarizability tensor of the neutral core whereas $\vec{E}$ is the local electric field given by the excess non-valence electron. This may belong to the same context with a recent report by the Wang group that the excess dipole-bound electron may play a significant role as the source of the intramolecular E-field. ${ }^{41}$ The simple physical model proposed here, though the quantum-mechanical correlation effect would be much more sophisticated, may be at least conceptually consistent with the correlation effect as far as the electron-radical interaction is concerned, as the electrons of the neutral core are allowed to be dynamically correlated with the excess non-valence electron by the mediation of the polarizability and the local electric field. F(Q) could be then re-written as follows.

$$
\mathrm{F}(\mathrm{Q})=\frac{1}{\epsilon_{i f}}\left\langle e_{f}\left|\frac{\partial \vec{\mu}_{e f f}}{\partial Q}\right| e_{i}\right\rangle=\frac{1}{\epsilon_{i f}}\left\langle e_{f}\left|\left(\frac{\partial \vec{\mu}_{0}}{\partial Q}+\frac{\partial \ddot{\alpha}}{\partial Q} \vec{E}\right)\right| e_{i}\right\rangle
$$

Here, $\vec{E}$ is assumed to be independent of Q. At the equilibrium position, as $\vec{E}$ heads from the neutral core to the dipole-bound electron, the neutral core is polarized so that the oxygen moiety is negatively charged whereas the opposite-positioned bromine moiety should be positively charged according to $\vec{\mu}_{\text {ind }}=\ddot{\alpha} \vec{E}$. The resultant induced-dipole, therefore, points the same direction as the permanent dipole (Figure 3a). And yet, the autodetachment process is not determined by the static property of the dipole. Rather, it is governed by the dynamic interplay between the instant changes of the permanent- and induced-dipoles with respect to the particular vibrational normal mode (Q). Accordingly, the directions of $\frac{\partial \vec{\mu}_{0}}{\partial Q}$ and $\frac{\partial \ddot{\alpha}}{\partial Q} \vec{E}$ vectors (the positive or negative slope with respect to Q) determine whether or not the correlation effect expedites or impedes the autodetachment process. Namely, if both $\frac{\partial \vec{\mu}_{0}}{\partial Q}$ and $\frac{\partial \ddot{\alpha}}{\partial Q} \vec{E}$ have the positive (or negative) slopes with respect to $Q$, then the autodetachment rate would increase, indicating that the correlation effect facilitates the autodetachment process. And yet, if the slope of $\frac{\partial \vec{\mu}_{0}}{\partial Q}$ is positive (or negative) while the slope of $\frac{\partial \ddot{\alpha}}{\partial Q} \vec{E}$ is negative (or positive), then the magnitude of the vector sum diminishes to give the decrease of the autodetachment rate. In this case, the autodetachment should be retarded due to the correlation effect. It should be noted that, when 
the dipole-bound electron is regarded as a point-charge lying on the molecule-fixed $z$-axis (Figures 1 and 3), all the in-plane vibrational modes of $\mathrm{PhO}^{-}$or 4-BrPhO- end up with the instant changes of dipole moments along the z-axis.

In order to testify the physical model, we have calculated the $\frac{\partial \mu_{0}}{\partial Q}$ and $\frac{\partial \alpha}{\partial Q}$ terms for the $v_{11}$ modes of 4 - $\mathrm{BrPhO}$ and $\mathrm{PhO}$, Figure $3 \mathrm{~b}$. For $\frac{\partial \mu_{0}}{\partial Q}, \mu_{0}$ is identical to the $z$-component of the permanent dipole moment $\left(\mu_{z}\right)$ (vide supra). Regarding $\frac{\partial \alpha}{\partial Q}$, the derivative of the isotropic polarizability $\left(\alpha_{\text {iso }}\right)$ or that of the polarizability along the molecular $z$-axis $\left(\alpha_{z z}\right)$ has been separately calculated with respect to the $v_{11}$ mode coordinate, and thus we denote $\alpha$ as either $\alpha_{\text {iso }}$ or $\alpha_{z z}$. It should be emphasized again that the $v_{11}$ modes of two different neutral cores of $4-\mathrm{BrPhO}$ and $\mathrm{PhO}$ have their own normal-mode characteristics in terms of the detailed nuclear displacements. Remarkably though, whereas the signs of $\frac{\partial \mu_{0}}{\partial Q}$ and $\frac{\partial \alpha}{\partial Q}$ are same for the $v_{11}$ mode of $\mathrm{PhO}$, it has been found that the slope of $\frac{\partial \mu_{0}}{\partial Q}$ has the opposite sign from that of $\frac{\partial \alpha}{\partial Q}$ for the $v_{11}$ mode of 4BrPhO. Apparently, the latter is the consequence from the reduction of the permanent dipole moment of $4-\mathrm{BrPhO}$ with the positive displacement of the $v_{11}$ mode whereas the polarizability along the z-axis instantly increases by the same displacement (Figure 3). Substitution of the electronegative $\mathrm{Br}$ atom on the para position should be responsible for the opposite behavior of the dipole-moment change with $v_{11}$, compared to that of PhO. Therefore, it is most likely that the correlation effect embodied in the charge-induced dipole interaction should impede the autodetachment of the $11^{\prime 1}$ mode of $4-\mathrm{BrPhO}^{-}$whereas it expedites that of the $11^{\prime 1}$ mode of $\mathrm{PhO}^{-}$ Though the quantitative comparison is nontrivial, it gives the rational explanation why the autodetachment rate could be exceptionally slow for the $11^{\prime 1}$ mode of $4-\mathrm{BrPhO}$. Notably, the magnitude of $\frac{\partial \alpha}{\partial Q}$ could be larger for $4-\mathrm{BrPhO}^{-}$compared to that of $\mathrm{PhO}^{-}$because of the lager polarizability of the former than the latter although the more sophisticated calculation is highly desirable (Supporting Information). 
(a)
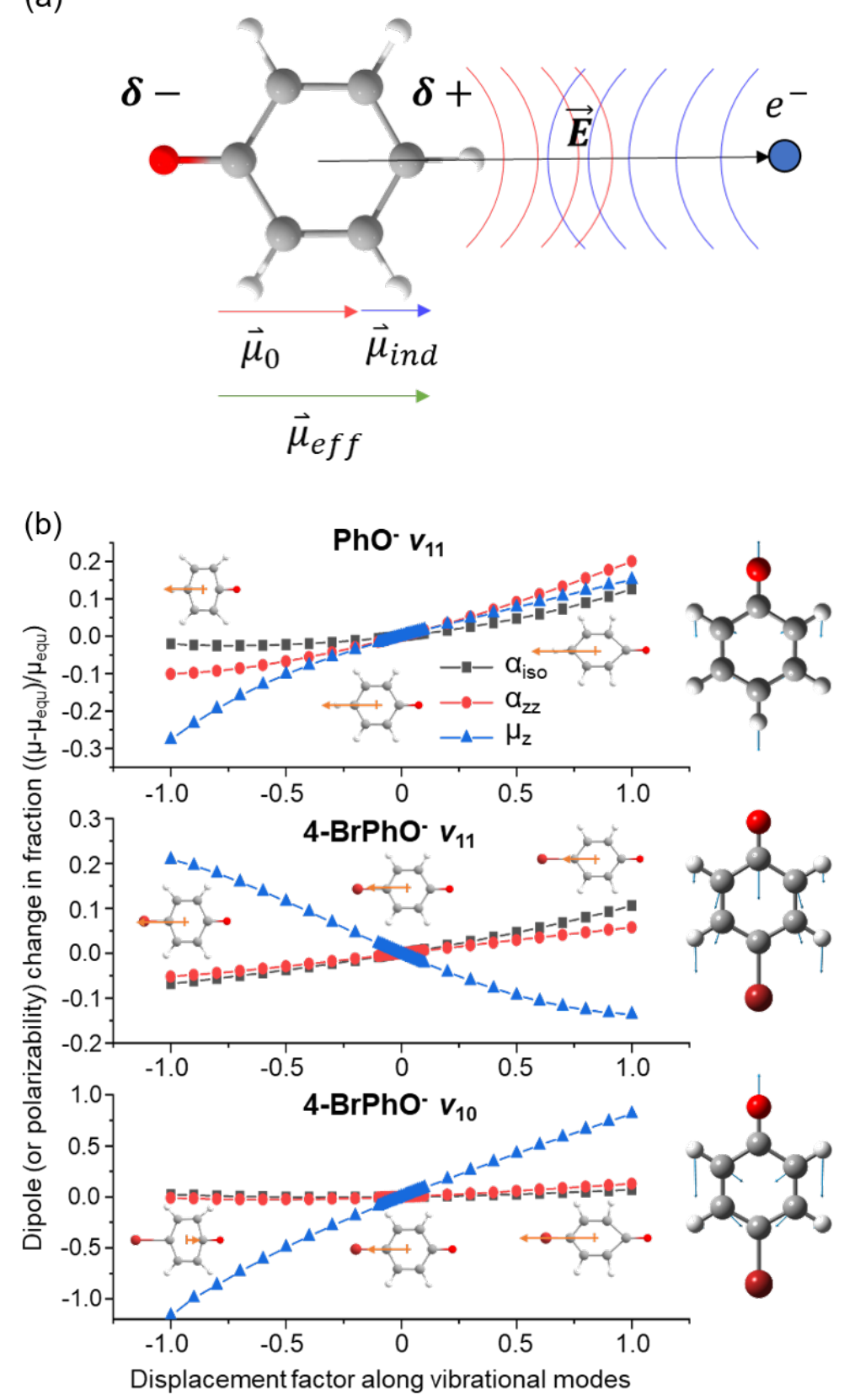

Figure 3. (a) Schematic diagram of the permanent dipole moment $\left(\vec{\mu}_{\mathbf{0}}\right)$ and induced dipole moment $\left(\overrightarrow{\boldsymbol{\mu}}_{\text {ind }}\right)$ in the presence of the electric field $(\overrightarrow{\boldsymbol{E}})$ given by the excess dipole-bound electron. (b) Changes of the dipole moments along the $z$-axis $\left(\mu_{z}\right)$, those of isotropic polarizabilities $\left(\alpha_{\text {iso }}\right)$, or those of polarizabilities along the diagonal $z$-axis $\left(\alpha_{z z}\right)$ for the $\mathrm{PhO}$ and $4-\mathrm{BrPhO}$ radicals calculated with respect to the $v_{11}$ and $v_{10}$ vibrational modes. Normal mode displacement vectors are denoted in the right panel. Dipole moments (orange arrow) at both ends of the normal mode displacement and equilibrium geometry are denoted. Note that direction of the dipole moment is denoted according to that of the actual electric dipole moment vector. 
The fast autodetachment rate $\left(\tau \sim 13.5 \mathrm{ps}\right.$ ) observed for the $20^{\prime 1} 30^{\prime 1}$ mode of $4-\mathrm{BrPhO}^{-}$is probably due to the cooperation effect of the combination mode in the wobbling motion as demonstrated previously for PhO- (Supporting Information). ${ }^{18}$ Regarding the $11^{\prime 2}$ overtone mode of $4-\mathrm{BrPhO}$, its lifetime of 82 ps is much longer than the lifetime of 12 ps measured for the $11^{\prime 2}$ mode of PhO- According to the derivative Franck-Condon factor in Eq. (2), the autodetachment rate of the overtone mode is anticipated to be $\sim 4$ times faster than that of the fundamental mode. ${ }^{18}$ In that sense, if the lifetime of the $11^{\prime 1}$ mode of $4-\mathrm{BrPhO}^{-}$is taken to be $2.54 \mathrm{~ns}$, the lifetime of $\sim 600$ ps is expected for the $11^{\prime 2}$ mode. In the same context, if the lifetime of $82 \mathrm{ps}$ is taken for the $11^{\prime 2}$ mode, the autodetachment lifetime of the $11^{\prime 1}$ mode is expected to be $\sim 330 \mathrm{ps,} \mathrm{which} \mathrm{is} \mathrm{already}$ quite long for the vibrational autodetachment lifetime. Therefore, although the lifetime measurement of $2.54 \mathrm{~ns}$ is subject to the further refinement, it seems to be quite certain that the autodetachment rate of the $11^{\prime 1}$ mode of $4-\mathrm{BrPhO}^{-}$is exceptionally slow. The fast autodetachment rate of the $10^{\prime 1}$ mode of $4-\mathrm{BrPhO}^{-}$with $\tau \sim 5.3$ ps is mainly attributed to the much stronger IR intensity of the $v_{10}$ mode which is $~ 30$ times larger than that of $v_{11}$, although the Fermi's golden rule could not give the quantitative explanation of the experiment (Supporting Information). It is interesting to note that the magnitude of $\frac{\partial \alpha}{\partial Q}$ is much smaller than that of $\frac{\partial \mu_{0}}{\partial Q}$ for the $10^{\prime 1}$ mode of 4-BrPhO-, Figure 3, suggesting that the dynamics of the corresponding mode is little influenced by the correlation effect.

Similar analysis has also been carried out for 4-ClPhO- For the Peak-III in Figure 1, the $19^{\prime} 120^{\prime} 1 / 11^{\prime 1}$ DBS band undergoes the autodetachment process via $-v_{19}$ (or $-v_{20}$ ) from the $19^{\prime} 120^{\prime 1}$ combination mode whereas the autodetachment from the $11^{\prime 1}$ mode is responsible for the $-v_{11}$ peak. The relative ratio of the former to the latter in the Peak-III is estimated to be $0.86: 0.14$. The peak-IV of $4-\mathrm{ClPhO}^{-}$(Figure 1) is assigned to the $11^{\prime 1} 19^{\prime} 120^{\prime 1} 111^{\prime 2}$ band. In the photoelectron spectrum, the photoelectron peak populated by the autodetachment via the $v_{20}$ or $v_{19}$ mode is found to be quite small. Similar to the case of Peak-III, the relative contribution of the $11^{\prime 1} 19^{\prime} 120^{\prime 1}$ and $11^{\prime 2}$ mode to the Peak-IV is estimated to be $0.86: 0.14$. Unfortunately, however, it turns out to 
be nontrivial to extract two different lifetimes from the transient of Peak-III or Peak-IV by the biexponential fit, mainly due to the relatively poor $\mathrm{S} / \mathrm{N}$ ratio, Figure 2. Instead, the single exponential fit to the experiment give the averaged autodetachment lifetime of $\sim 548 \pm 108$ or 50.0 \pm 8.5 ps for the Peak-III or Peak-IV, respectively. Overall, the autodetachment rate of the 4-ClPhODBS is also estimated to be quite slow compared to that of $\mathrm{PhO}^{-}$, indicating that the correlation effect on the electron-binding dynamics could also be quite significant in 4-ClPhO- It should be emphasized that the autodetachment dynamics is expected to be strongly dependent on the individual chemical systems, and thus the theoretical analyses for individual chemical systems should be carried out case by case.
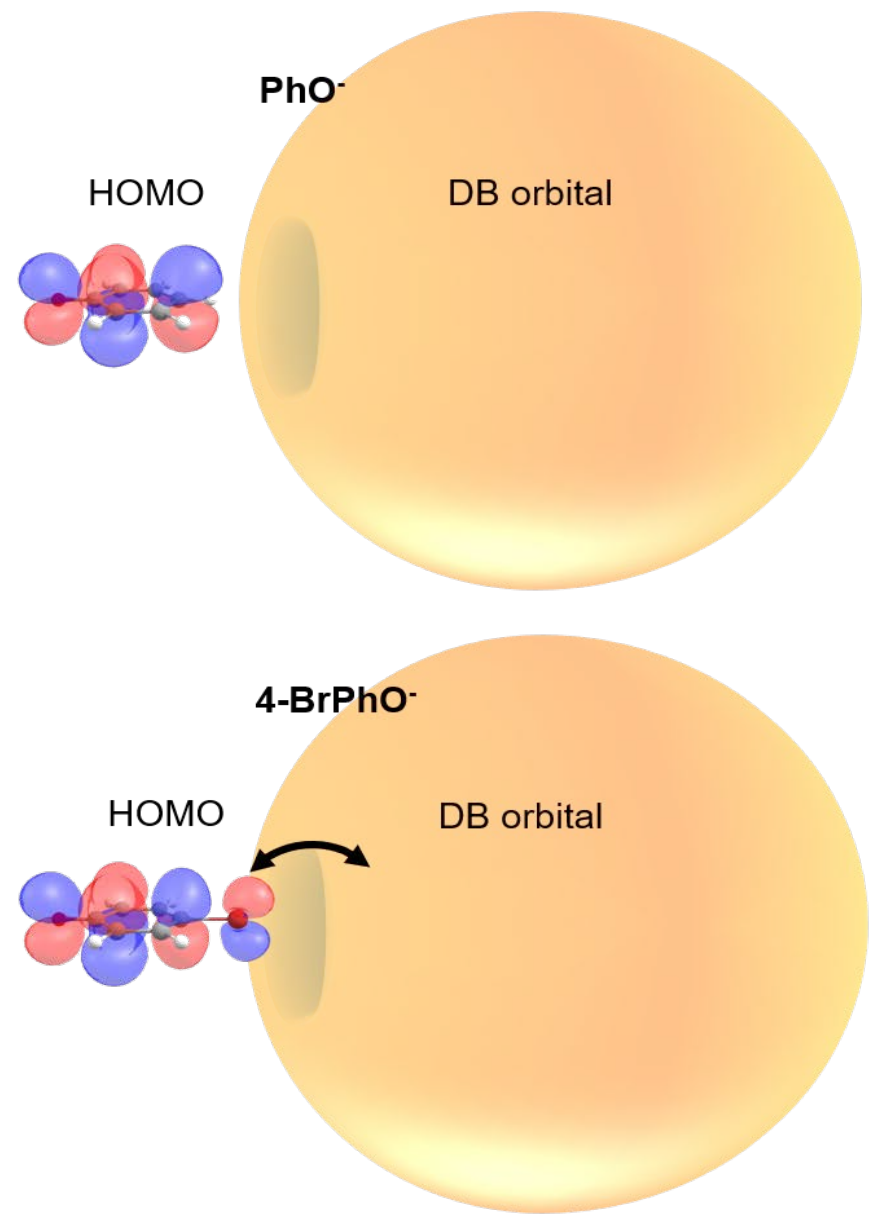

Figure 4. Schematic diagram of the correlation effect between the half-filling HOMO and DB orbital in the DBS electron configuration. Orbital robes on the $\mathrm{Br}$ atom in the para-position may enhance the correlation effect between the HOMO and DB orbital, giving the slow autodetachment rate. 
It is interesting to note that the highest occupied molecular orbital (HOMO) of the 4$\mathrm{BrPhO}^{-}$or 4-ClPhO- is delocalized over the entire body including the $p$-type lobe of the halogen atomic moiety at the positive end of the dipole (Figure 4). The excess non-valence electron is diffuse and polarizable whereas it could exert the local electric field to influence the electrons in the neutral core.$^{41}$ In this aspect, the relative geometrical orbital arrangements of the neutral-core with respect to the excess electron of which its own arrangement is given by the molecular geometry could be quite critical in the role of the correlation effect in static and dynamic properties of the DBS, which is subject to further investigation in the near future.

In summary, the conventional Fermi's golden rule based on the simple monopole-dipole interaction potential may not be universal in explaining the autodetachment dynamics of the dipole bound state. Exceptionally slow autodetachment dynamics observed in the DBS of 4$\mathrm{BrPhO}^{-}$and 4- $\mathrm{ClPhO}^{-}$have been analyzed by including the electron correlation effect in the form of the induced dipole-moment. The induced-dipole is dynamic in nature as it is supposed to be the consequence from the local field given by the interaction between polarizable excess nonvalence electron and valence electrons of the neutral-core. A simple physical model containing the correlation effect, adapted from the Fermi's golden rule, turns out to be quite useful in the qualitative explanation of the experiment. The exceptionally slow autodetachment rate of the $11^{\prime 1}$ mode of 4-BrPhO- DBS may originate from the fact that the derivative of the permanent dipole has the directional property of the opposite sign from that of the polarizability with respect to the corresponding normal mode displacement. In this case, the correlation effect embodied in the physical model impedes the autodetachment process. On the other hand, when the derivative of the permanent dipole and that of the polarizability are same in their directional properties, the autodetachment is expected to be expedited as demonstrated in the case of the $11^{\prime 1}$ mode of PhOThis should belong to the case where the autodetachment is facilitated by the correlation effect. The autodetachment process is strongly mode-dependent and also it is highly dependent on the individual chemical system. In this circumstance, the real-time investigation of the autodetachment dynamics, as manifested in this report, could unravel different aspects of the 
underlying physical principles behind the nature of the non-valence bound states, challenging the sophisticated theoretical analysis in the near future.

\section{Methods}

Details of the electrospray ionization-photoelectron imaging (ESI-PEI) apparatus have been described elsewhere. ${ }^{42} 1 \mathrm{mM}$ concentration of the phenol and $4-\mathrm{XPhOH}(\mathrm{X}=-\mathrm{Cl},-\mathrm{Br}, \mathrm{TCI}$ chemicals) were dissolved in the 9:1 methanol/water solution without further purifications. Anions were generated from the home-made ESI source and de-solvated by the dual-stage ion funnel (IF141, Masstech Inc.). Target anions were transferred into the cryogenic ( 8 K) Paul ion trap where they were internally cooled with the buffer gas of the $(4: 1) \mathrm{He} / \mathrm{H}_{2}$ mixture. The cryogenically-cooled anions were then extracted and accelerated to the velocity map electron imaging (VMI) apparatus. Photoelectrons ejected by the interaction with the picosecond laser pulses were detected by the microchannel plates backed by the phosphor screen prior to be recorded by a charge-coupled device (CCD) camera. Picosecond laser pulses were generated from the picosecond Ti:Sapphire regenerative amplifier (Legend Elite-P, Coherent) seeded by the femtosecond Ti:Sapphire oscillator (Vitra-T-HP, Coherent). One half of the fundamental output of the regenerative amplifier was tuned by an optical parametric amplifier (TOPAS-800, Light Conversion) for the pump laser pulse, while the other half of the fundamental was used as the probe laser pulse. Time-resolved photoelectron images were obtained by scanning the delay times between the pump and probe pulses using a couple of retro-reflectors on the optical delay stage (DDS220, Thorlabs). Photoelectron images were reconstructed by the BASEX ${ }^{43}$ or polar onion peeling (POP) programs. ${ }^{44}$ 


\section{Associated Content}

Supporting Information

Details of the photoelectron imaging deconvolution, vibrational modes of each molecule, and dipole or polarizability change upon the associated molecular vibration were described.

\section{Author Information}

Corresponding author

Sang Kyu Kim

*sangkyukim@kaist.ac.kr

ORCID

Do Hyung Kang: 0000-0003-4774-9062

Jinwoo Kim: 0000-0003-2967-6904

Sang Kyu Kim: 0000-0003-4803-1327

Acknowledgement: This work was supported by the National Research Foundation of Korea (2018R1A2B3004534, 2019K1A3A1A14064258, and 2019R1A6A1A10073887).

\section{References}

1. E. Fermi and E. Teller, Phys. Rev., 1947, 72, 399-408.

2. K. R. Lykke, R. D. Mead and W. C. Lineberger, Phys. Rev. Lett., 1984, 52, 2221-2224.

3. R. N. Compton and N. I. Hammer, Multipole-bound Molecular Anion, New York, 2001.

4. K. D. Jordan and F. Wang, Annu. Rev. Phys. Chem., 2003, 54, 367-396.

5. G.-Z. Zhu and L.-S. Wang, Chem. Sci., 2019, 10, 9409-9423.

6. J. N. Bull, C. S. Anstöter and J. R. R. Verlet, Nat. Commun., 2019, 10, 5820.

7. J. N. Bull, C. W. West and J. R. R. Verlet, Chem. Sci., 2016, 7, 5352-5361.

8. V. F. Sidorkin, E. F. Belogolova, E. P. Doronina, G. Liu, S. M. Ciborowski and K. H. Bowen, J. Am. 
Chem. Soc., 2020, 142, 2001-2011.

9. T. Sommerfeld, Phys. Chem. Chem. Phys., 2002, 4, 2511-2516.

10. T. Sommerfeld, J. Phys.: Conf. Ser., 2005, 4, 245-250.

11. D. Tripathi and A. K. Dutta, J. Phys. Chem. A, 2019, 123, 10131-10138.

12. J. R. R. Verlet, C. S. Anstöter, J. N. Bull and J. P. Rogers, J. Phys. Chem. A, 2020, 124, 3507-3519.

13. R. N. Compton, H. S. Carman, C. Desfrançois, H. Abdoul-Carime, J. P. Schermann, J. H. Hendricks, S. A. Lyapustina and K. H. Bowen, J. Chem. Phys., 1996, 105, 3472-3478.

14. G. Liu, S. M. Ciborowski, J. D. Graham, A. M. Buytendyk and K. H. Bowen, J. Chem. Phys., 2020, $153,044307$.

15. D. H. Kang, J. Kim and S. K. Kim, J. Phys. Chem. Lett., 2021, DOI: 10.1021/acs.jpclett.1c01789, 63836388.

16. X.-B. Wang and L.-S. Wang, Rev. Sci. Instrum., 2008, 79, 073108.

17. H.-T. Liu, C.-G. Ning, D.-L. Huang, P. D. Dau and L.-S. Wang, Angew. Chem., Int. Ed., 2013, 52, 89768979 .

18. D. H. Kang, S. An and S. K. Kim, Phys. Rev. Lett., 2020, 125, 093001.

19. O. H. Crawford, Mol. Phys., 1971, 20, 585-591.

20. W. R. Garrett, Chem. Phys. Lett., 1970, 5, 393-397.

21. W. R. Garrett, Phys. Rev. A, 1971, 3, 961-972.

22. C.-H. Qian, G.-Z. Zhu and L.-S. Wang, J. Phys. Chem. Lett., 2019, 10, 6472-6477.

23. C. Desfrançois, H. Abdoul-Carime, N. Khelifa and J. P. Schermann, Phys. Rev. Lett., 1994, 73, 24362439.

24. M. Gutowski, P. Skurski, A. I. Boldyrev, J. Simons and K. D. Jordan, Phys. Rev. A, 1996, 54, 19061909.

25. M. Gutowski, K. D. Jordan and P. Skurski, J. Phys. Chem. A, 1998, 102, 2624-2633.

26. H. Abdoul-Carime and C. Desfrançois, Eur. Phys. J. D, 1998, 2, 149-156.

27. T. Sommerfeld, A. DeFusco and K. D. Jordan, J. Phys. Chem. A, 2008, 112, 11021-11035.

28. T. Sommerfeld, J. Phys. Chem. A, 2008, 112, 11817-11823.

29. H. Hao, J. Shee, S. Upadhyay, C. Ataca, K. D. Jordan and B. M. Rubenstein, J. Phys. Chem. Lett., 2018, 9, 6185-6190.

30. K. A. Peterson and M. Gutowski, J. Chem. Phys., 2002, 116, 3297-3299. 
31. D.-F. Yuan, Y.-R. Zhang, C.-H. Qian, Y. Liu and L.-S. Wang, J. Phys. Chem. A, 2021, 125, 2967-2976.

32. D.-F. Yuan, Y. Liu, C.-H. Qian, Y.-R. Zhang, B. M. Rubenstein and L.-S. Wang, Phys. Rev. Lett., 2020, $125,073003$.

33. Y. Lu, R. Tang and C. Ning, J. Phys. Chem. Lett., 2021, 12, 5897-5902.

34. C. Desfrançois, Phys. Rev. A, 1995, 51, 3667-3675.

35. D. M. Neumark, K. R. Lykke, T. Andersen and W. C. Lineberger, J. Chem. Phys., 1985, 83, 4364-4373.

36. K. Yokoyama, G. W. Leach, J. B. Kim, W. C. Lineberger, A. I. Boldyrev and M. Gutowski, J. Chem. Phys., 1996, 105, 10706-10718.

37. K. Yokoyama, G. W. Leach, J. B. Kim and W. C. Lineberger, J. Chem. Phys., 1996, 105, 10696-10705.

38. D. O'Neal and J. Simons, J. Phys. Chem., 1989, 93, 58-61.

39. C. S. Anstöter, G. Mensa-Bonsu, P. Nag, M. Ranković, R. Kumar T. P, A. N. Boichenko, A. V. Bochenkova, J. Fedor and J. R. R. Verlet, Phys. Rev. Lett., 2020, 124, 203401.

40. J. Simons, J. Phys. Chem. A, 2020, 124, 8778-8797.

41. D.-F. Yuan, Y. Liu, C.-H. Qian, G. S. Kocheril, Y.-R. Zhang, B. M. Rubenstein and L.-S. Wang, J. Phys. Chem. Lett., 2020, 11, 7914-7919.

42. D. H. Kang, J. Kim, M. Cheng and S. K. Kim, J. Phys. Chem. Lett., 2021, 12, 1947-1954.

43. V. Dribinski, A. Ossadtchi, V. A. Mandelshtam and H. Reisler, Rev. Sci. Instrum., 2002, 73, 2634-2642.

44. G. M. Roberts, J. L. Nixon, J. Lecointre, E. Wrede and J. R. R. Verlet, Rev. Sci. Instrum., 2009, 80, 053104 . 